\title{
Immunomodulatory capacity of human mesenchymal stem cells isolated from adipose tissue, dental pulp, peripheral blood and umbilical cord Wharton's jelly
}

\author{
DRENKA TRIVANOVIĆ, SLAVKO MOJSILOVIĆ, VESNA ILIĆ, JELENA KRSTIĆ, \\ ALEKSANDRA JAUKOVIĆ, IVANA OKIĆ-ĐORĐEVIĆ, JUAN FRANCISCO SANTIBANEZ, \\ GORDANA JOVČIĆ, DIANA BUGARSKI
}

Laboratory for Experimental Hematology and Stem Cells, Institute for Medical Research, University of Belgrade, Belgrade, Serbia

\begin{abstract}
Mesenchymal stem cells (MSCs), beside regenerative potential, possess immunomodulatory properties and their use in managing immune-mediated diseases is intensively studied. We analyzed the effects of MSCs isolated from human adipose tissue (AT-MSCs), dental pulp (DP-MSCs), peripheral blood (PB-MSCs) and umbilical cord Wharton's jelly (UC-MSCs), on the proliferation of allogeneic peripheral blood mononuclear cells (PBMCs). While only AT-MSCs functioned as alloantigen presenting cells, proliferation of PBMCs in response to a phytohemagglutinin (PHA) and alloantigens in mixed lymphocytes reaction (MLR) was inhibited by all MSCs in a cell concentration-dependent manner. Conditioned medium (CM) derived from DP-MSCs, PB-MSCs and UC-MSCs, suppressed the baseline, PHA- and alloantigens-mediated proliferation of PBMC, whereas AT-MSCs-derived CM inhibited MLR, but failed to suppress the spontaneous and PHA-induced PBMCs proliferation. Differences between MSC types were observed in expression of genes related to immunomodulation, including human leukocyte antigens (HLA)-A, HLA-DR, HLA-G5, interleukin 6 (IL)-6, transforming growth factor (TGF)- $\beta$, cyclooxygenase-2 (COX-2) and indoleamine 2,3-dioxygenase (IDO-1), under basal conditions, as well as in response to proinflammatory cytokines, interferon (IFN)- $\gamma$ and tumor necrosis factor $\alpha(T N F)-\alpha$. While AT-MSCs showed a positive constitutive expression of almost all tested genes that was augmented in response to IFN- $\gamma$ and TNF- $\alpha$, only combined cytokine treatment increased HLA-A, COX2 and IL-6 mRNA expression in DP-MSCs and slightly stimulated the expression of HLA-G and TGF- $\beta$ in UC-MSCs. Although MSCs from different tissues showed similar potential to suppress proliferation of PBMCs, heterogeneity in the expression of genes related to immunomodulation emphasizes the importance of investigating the role of specific molecular mechanisms in the regulation of immunomodulatory activity of MSCs.
\end{abstract}

Key words: immunomodulation, mesenchymal stem cells, PBMCs, proinflammatory.

(Centr Eur J Immunol 2013; 38 (4): 421-429)

\section{Introduction}

Mesenchymal stem cells (MSCs) have been described as undifferentiated multipotent cells with the ability of both self-renewing and differentiation into a wide range of cells of mesenchymal lineages, such as osteoblasts, chondrocytes, adipocytes, (bone marrow) stromal cells, fibroblasts and tendons, but also of ectodermal and endodermal types (hepatocytes, neural cells, lung cells, and endothelial cells) [1,2]. MSCs were originally isolated from bone marrow [3] but cells with generally similar, although not always identical properties were found in diverse adult tissues (peripheral blood, adipose tissue, trabecular bone, synovial membrane, dental pulp, etc.), as well as in birth-associated tissues (placenta, amnion, umbilical cord, and cord blood) [1,2].

For the last two decades MSCs have gained much attention because of their regenerative, reparative and angiogenic properties $[1,4]$. Additional therapeutic potential of MSCs is related to their capacity to modulate the 
immune response exerting effects on cells of both innate and adaptive immunity $[2,5,6]$. The immunomodulatory effects of MSCs include suppression of T cell proliferation, induction of regulatory $\mathrm{T}$ cells, suppression of $\mathrm{B}$ cell proliferation and their terminal differentiation, inhibition of NK cell function, as well as inhibition of differentiation and expression of stimulatory molecules on dendritic cells [2]. However, the mechanisms by which MSCs exert their immunomodulatory activity are only partly understood, although it is likely that mechanisms involving both soluble factors and cell-cell contacts are involved. There is growing evidence that key relevant mediators of MSC-mediated immunomodulation include but are not limited to: transforming growth factor- $\beta$ (TGF- $\beta$ ), cyclooxygenase- 1 (COX-1) and COX-2, which synthesizes prostaglandin E2 (PGE2), indoleamine 2,3 deoxygenase (IDO), nitric oxide (NO), human leukocyte antigen (HLA)-G5, interleukin 6 (IL-6), IL-10 [2, 6]. Another open issue is the relation of the MSCs immunomodulatory phenotype to the microenvironmental context, since recent data indicated that both MSCs behavior and function are influenced by cytokines and other factors secreted or accumulated in the microenvironment, especially within the inflamed tissue [7-9].

MSCs of diverse origin are currently available, and each type has its own strength and shortcomings. Our group has recently isolated MSCs from four different human tissues: peripheral blood (PB), umbilical cord Wharton's jelly (UC), adipose tissue (AT), and dental pulp (DP) from deciduous teeth, [10, 11, our submitted manuscript], whose common feature is that all are discarded after routine medical interventions and therefore are a readily available source for MSCs isolation. It is well documented that MSCs phenotype, proliferative, clonogenic and differentiation potential, as well as the immunomodulatory properties are influenced by tissue origin, donor-related health condition and age, isolation technique, cultivation, propagation, and storage conditions [12-14]. Therefore, comparative evaluation of MSCs from different sources is needed to address the existence of possible differences. In this study, with an aim to determine how tissue origin and/or proinflammatory cytokines influence the immunomodulatory phenotype, we have compared MSCs obtained from peripheral blood, umbilical cord Wharton's jelly, adipose tissue and deciduous teeth-dental pulp by evaluating their in vitro immunomodulatory capacities, as well as the gene expression of several molecules that are of importance in determination of the nature of the response generated by MSCs.

\section{Material and methods}

\section{MSCs isolation and culture}

Cell samples were obtained in accordance with the ethical standards of the local ethical committee and the Declaration of Helsinki, and after providing informed consent of study subjects. MCSs from human dental pulp of deciduous teeth (DP-MSCs), peripheral blood (PB-MSCs), umbilical cord Wharton's jelly (UC-MSCs), as well as from adipose tissue (AT-MSCs), were isolated and their MSCs identity confirmed as previously described [10, 11].

After isolation, AT-MSCs, PB-MSCs, and UC-MSCs were further cultivated in the growth medium (GM) consisting of Dulbecco's Modified Eagle's Medium (DMEM, Sigma-Aldrich) supplemented with $10 \%$ Fetal Bovine Serum (FBS) and Penicillin/Streptomycin (all from PAA, Linz, Austria), whereas DP-MSCs were cultured in GM with $200 \mu \mathrm{M}$ ascorbic acid-2-phosphate (Sigma-Aldrich, St. Louis, MO, USA). Cells were seeded in $25 \mathrm{~cm}^{2}$ plastic tissue culture flasks at a concentration of $1 \times 10^{4} \mathrm{cells} / \mathrm{cm}^{2}$ and incubated in a humidified atmosphere at $37^{\circ} \mathrm{C}$ and $5 \% \mathrm{CO}_{2}$ with the medium replaced every 2-3 days. After achieving 80-90\% of confluence, MSCs were detached by $0.25 \%$ trypsin-EDTA solution (PAA) and replated at the same cell density. All experiments were performed using MSCs between passages 4 and 6.

\section{PBMCs isolation}

Peripheral blood mononuclear cells (PBMCs) were isolated from buffy coats of healthy donors by density gradient centrifugation using Lymphoprep medium (PAA).

\section{Conditioned media preparation}

The conditioned medium (CM) derived from MSCs was obtained as described previously [15]. In brief, after reaching $80 \%$ of confluence, the cell culture medium was replaced by fresh GM. After additional $48 \mathrm{~h}$ of cultivation, CM-MSCs were collected. The CM-MSCs, containing factors constitutively secreted by unstimulated MSCs, were centrifuged for $10 \mathrm{~min}$ at $500 \times \mathrm{g}$ and aliquots stored at $-70^{\circ} \mathrm{C}$ until use.

\section{In vitro assays for testing MSCs modulatory effects on PBMCs proliferation}

\section{One-way mixed lymphocyte reaction (MLR)}

To determine if MSCs function as alloantigens presenting cells one-way MLR was applied. MSCs at concentrations of $1 \times 10^{4}$ or $1 \times 10^{3}$ were plated in 96-well plates in $\mathrm{GM}$ and allowed to adhere at $37^{\circ} \mathrm{C}$ in $5 \% \mathrm{CO}_{2}$. After overnight incubation, MSCs were treated with Mitomycin C (25 $\mu \mathrm{g} / \mathrm{ml})$ (Applichem, Darmstadt, Germany) for $30 \mathrm{~min}$ at $37^{\circ} \mathrm{C}$. After washing MSCs with phosphate buffered saline (PBS, PAA, Linz), GM (200 ml/well) was added along with freshly isolated responder PBMCs $\left(1 \times 10^{5}\right.$ cells $)$. Cultures of PBMCs seeded alone were used as controls. On day 2, cultures were pulsed with $1 \times 10^{-5} \mathrm{M}$ BrdU (Sigma-Aldrich) for the final $24 \mathrm{~h}$. The level of proliferation, i.e. the level of BrdU incorporation, was measured using a modified method [16]. In brief, ethanol/ $\mathrm{HCl}$ fixed cells were incubated 
with mouse anti-BrdU IgG antibody (Sigma-Aldrich), and then with peroxidase conjugated anti-mouse IgG antibodies (Pierce Biotechnology, Rockford, IL, USA). After addition of peroxidase substrate, the level of proliferation was estimated by measuring the absorbance at $450 \mathrm{~nm}$. All experiments were performed in triplicate.

\section{Mitogen-stimulated PBMCs proliferation}

To estimate the effects of MSCs on phytohaemagglutinin (PHA)-stimulated proliferative response of allogeneic PBMCs, freshly isolated responder PBMCs $\left(1 \times 10^{5} /\right.$ well $)$, stimulated with $2.5 \mu \mathrm{g} / \mathrm{ml}$ PHA (INEP, Zemun, Serbia) were added in GM to MSCs cultures, previously seeded and treated with Mitomycin C, as described above. Cultures of unstimulated and PHA-stimulated PBMCs seeded without MSCs were used as controls. On day 2, cultures were pulsed with $1 \times 10^{-5} \mathrm{M}$ BrdU for the final $24 \mathrm{~h}$ of culture. The level of proliferation was measured as described above. All experiments were performed in triplicate.

\section{Two-way MLR}

To assess the effects of MSCs on two-way MLR (as a third part in MLR), PBMCs of unrelated donors $\left(1 \times 10^{5}\right.$ cells of each) were co-cultured in GM with MSCs previously seeded and treated with Mitomycin $\mathrm{C}$ as described above. Co-cultures of responder PBMCs and allogeneic stimulator PBMCs without MSCs were used as controls. On day 5, cultures were pulsed with $1 \times 10^{-5} \mathrm{M} \mathrm{BrdU}$ for the final $24 \mathrm{~h}$. The level of proliferation was measured as described above. All experiments were performed in triplicate.

\section{Conditioned media experiments}

The effects of MSCs-derived soluble products were assessed in both PHA-stimulated PBMC proliferation and the one- and two-way MLR. Briefly, $1 \times 10^{5}$ responder PBMCs, incubated in the presence or absence of $2.5 \mu \mathrm{g} / \mathrm{ml}$ PHA, or with $1 \times 10^{5}$ PBMCs of unrelated donors for the MLR, were exposed to GM supplemented with $20 \%$ CM of each MSCs type for 3 or 6 days and cultured in triplicate at $37^{\circ} \mathrm{C}, 5 \%$ of $\mathrm{CO}_{2}$ before the MTT assay was carried out. The experiments were performed at least three times and cultures without $\mathrm{CM}$ were used as controls. The PBMC proliferation rate was determined by MTT (3-(4,5-Dimethylthiazol-2-yl)-2,5-diphenyltetrazolium bromide) assay. Three hours after adding 0.5 $\mathrm{mg} / \mathrm{ml}$ MTT (Sigma-Aldrich), the formazan crystals were dissolved in $10 \%$ SDS with $0.01 \mathrm{~N} \mathrm{HCl}$ and absorbance read at $540 \mathrm{~nm}$.

\section{Reverse transcription-polymerase chain reaction (RT-PCR)}

For gene expression analyses, MSCs treated or not with recombinant human IFN- $\gamma(50 \mathrm{ng} / \mathrm{ml})$ and/or TNF- $\alpha$ (20 ng/ml) (all from R\&D Systems, Minneapolis, MN, USA) for 7 days were used. In a separate experiment, subconfluent MSCs were co-cultured for 2 days, with $1 \times 10^{6}$ allogeneic unstimulated or PHA-stimulated PBMCs. Cells grown only in GM were used as controls. Total RNA from MSCs was extracted using TRIzol (Invitrogen, Carlsbad, California, USA) and complementary DNA generated by the RevertAid TM Hminus First Strand cDNA Synthesis Kit (Fermentas, Life Science, Glen Burnie, MD, USA) using oligo (dT) as a primer. Glyceraldehyde 3-phosphate dehydrogenase (GAPDH) was used as a control for the amount of cDNA present in each sample. The primers sets were all purchased from Invitrogen, Carlsbad, CA (Table 1). PCR bands were quantified by densitometry using the ImageMaster TotalLab v1.11 software (Amersham Pharmacia Biotech, Uppsala, Sweden). The expression level of each gene was normalized to GAPDH and set as 1 for control culture. The relative expression in stimulated MSCs was expressed as "fold change" compared to the control.

Table 1.

\begin{tabular}{|c|c|c|c|c|c|}
\hline Primer & Forward sequence 5'-3', & Reverse sequence 5'-3' & $\begin{array}{c}\text { Annealing } \\
\text { temperature } \\
\left({ }^{\circ} \mathrm{C}\right)\end{array}$ & Cycles & $\begin{array}{l}\text { Product size } \\
\text { (bp) }\end{array}$ \\
\hline GAPDH & ACCACAGTCCATGCCATCAC & TCCACCACCCTGTTGCTGTA & 52 & 35 & 452 \\
\hline HLA-A & GACGACACGCAGTTCGTGC & CATGTCCGCCGCGGTCCAA & 49 & 31 & 331 \\
\hline HLA-G5 & GGAAGAGGAGACACGGAACA & CCTTTTCAATCTGAGCTCTTCTTT & 47 & 40 & 771 \\
\hline HLA-DR $\alpha$ & CGAGTTCTCTATCTGAATCCTG & GTTCTGCTGCATTGCTTTTGC & 52 & 33 & 644 \\
\hline IL-6 & ATGAACTCCTTCTCCACAAG & AGAGCCCTCAGGCTGGACTG & 53 & 35 & 626 \\
\hline $\mathrm{COX} 2$ & СCTTCCTCCTGTGCCTGATG & CTGGCCCTCGCTTATGATCT & 59 & 35 & 203 \\
\hline IDO-1 & ATCACCATGGCATATGTGTGGG & GTGAAACACTTGAAGGGCTTTCTC & 51 & 33 & 390 \\
\hline TGF- $\beta$ & GGGACTATCCACCTGCAAGA & CCTCCTTGGCGTAGTAGTCG & 50 & 33 & 239 \\
\hline
\end{tabular}


A

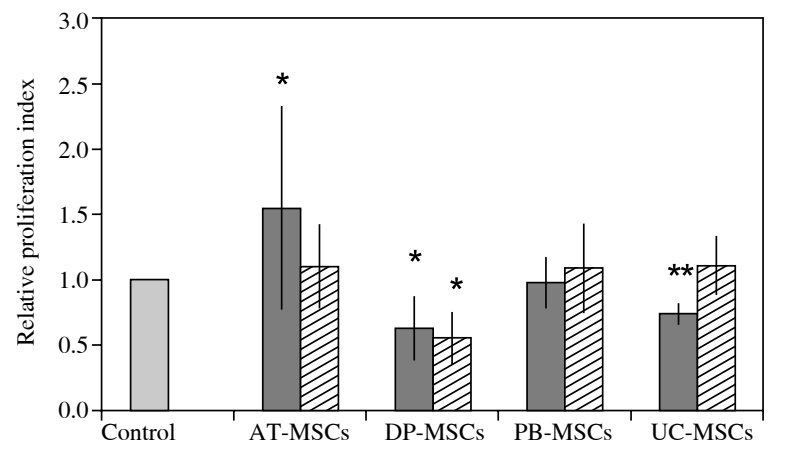

B

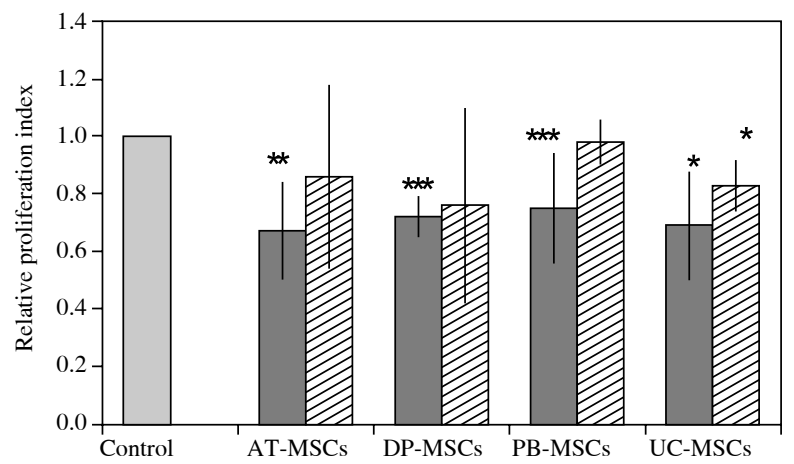

$\mathrm{C}$

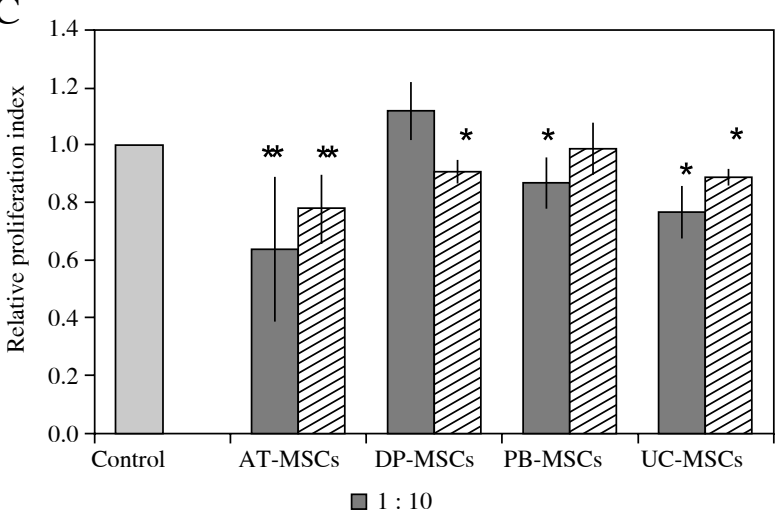

$\square 1: 100$

Fig. 1A-C. Influence of AT-MSCs, DP-MSCs, PB-MSCs, and UC-MSCs on spontaneous (A), mitogen- (B) or alloantigen-induced (C) PBMCs proliferation. A) The alloantigen presenting function of AT-MSCs, DP-MSCs, PB-MSCs, and UC-MSCs. Allogeneic PBMCs $\left(1 \times 10^{5}\right)$ were co-cultured for three days in GM with Mitomycin C-pretreated MSCs at $1: 100$ and $1: 10 \mathrm{MSCs} / \mathrm{PBMCs}$ ratios. B) The modulatory effect of AT-MSCs, DP-MSCs, PB-MSCs, and UC-MSCs on PHA-stimulated proliferation of PBMCs. Allogeneic PBMCs $\left(1 \times 10^{5}\right)$ stimulated with $2.5 \mu \mathrm{g} / \mathrm{ml}$ PHA were co-cultured for three days in GM with Mitomycin C-pretreated MSCs at $1: 100$ and $1: 10 \mathrm{MSCs} / \mathrm{PBMCs}$ ratios. C) The AT-MSCs, DP-MSCs, PB-MSCs, and UC-MSCs as third part modulators of two-way MLR. PBMCs $\left(1 \times 10^{5}\right)$ of two unrelated donors were cultivated for six days in GM with Mitomycin C-pretreated MSCs at $1: 100$ and $1: 10 \mathrm{MSCs} / \mathrm{PBMCs}$ ratios. The proliferation of PBMCs was determined by BrdU incorporation. The allogeneic PBMCs cultured without MSCs were used as controls and the proliferation value in control cultures were expressed as equal to 1 . The data are mean $\pm \mathrm{SD}$ of three to six independent experiments performed in triplicate. Results are presented as a relative proliferation index where the level of proliferation of PBMCs in allogeneic MLR was set at 1 $* p<0.05, * * p<0.01$ and $* * * p<0.001$ compared to the corresponding controls

\section{Statistical analyses}

The results are presented as the mean \pm standard deviation (SD). The statistical significance of differences between two groups was determined by the two-tailed Student's $t$-test. Differences with $P$-values of $<0.05$ were considered significant.

\section{Results}

\section{Immunomodulatory properties of different MSC populations}

First, we analyzed if MSCs can act as alloantigens presenting cells. Different concentrations of MSCs inactivated by Mitomycin C were co-cultured with responder allogeneic PBMCs before the resulting PBMCs proliferation was assessed. As shown in Fig. 1A, only AT-MSCs stimulated the proliferation of allogeneic PBMCs, and just at the $1: 10$ AT-MSCs to PBMCs ratio. On the other hand, PB-MSCs did not exert any effect, while DP-MSCs and UC-MSCs even suppressed the proliferation of PBMCs in a MSC concentration-dependent manner.

Next, we evaluated the effect of MSCs on the mitogen-mediated lymphocyte proliferation and obtained results demonstrated that all tested MSCs inhibited the proliferation of PBMCs in response to PHA treatment in a MSC concentration-dependent manner (Fig. 1B). The UC-MSCs significantly inhibited the PBMC proliferation at both cell concentrations tested, i.e. at MSCs/PBMCs ratio of $1: 10$ and $1: 100$, whereas AT-MSCs, DP-MSCs, 
A

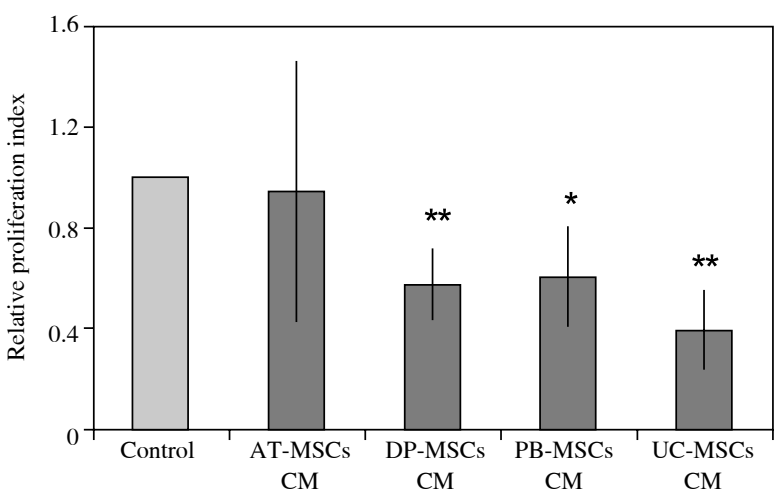

B

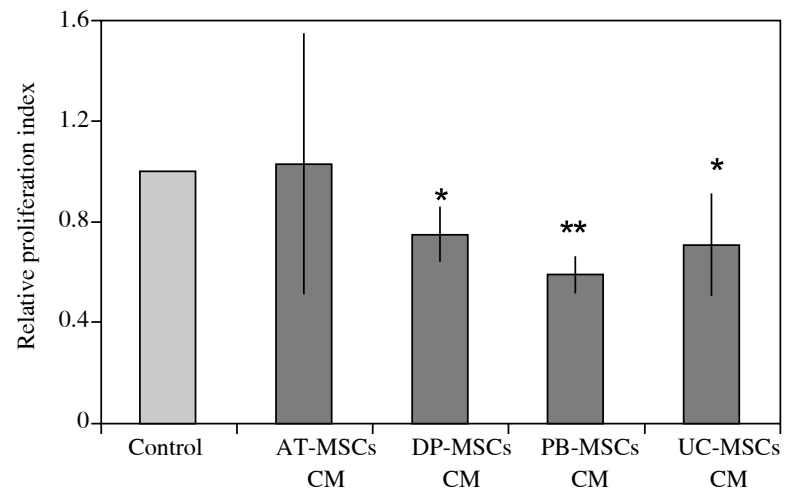

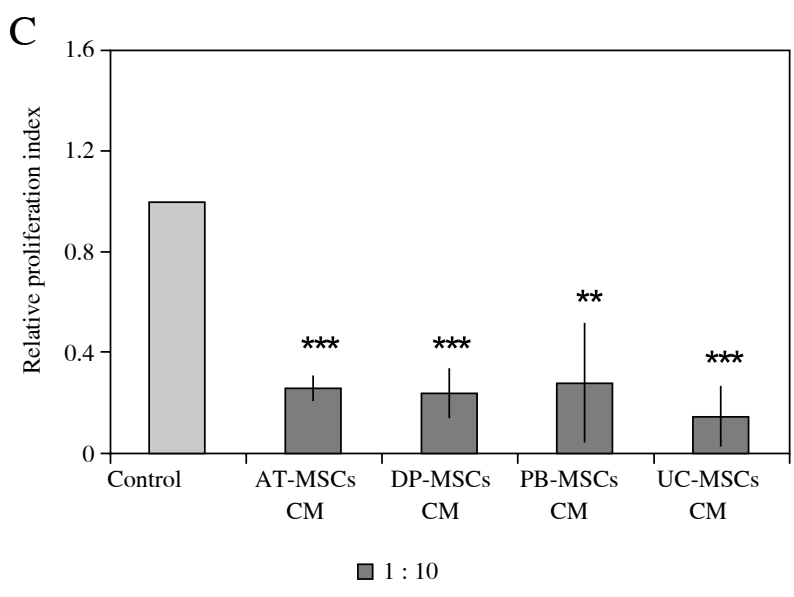

$\square 1: 100$

Fig. 2. Effects of CM derived from AT-MSCs, DP-MSCs, PB-MSCs, and UC-MSCs on spontaneous (A), mitogen- (B) or alloantigen-induced (C) PBMCs proliferation. Allogeneic responder PBMCs $\left(1 \times 10^{5}\right)$, incubated in the absence of mitogen A) or presence of $2.5 \mu \mathrm{g} / \mathrm{ml}$ PHA B), or with PBMCs $\left(1 \times 10^{5}\right)$ of unrelated donors C), were exposed to GM supplemented with 20\% CM of each MSCs type for 3 or 6 days. PBMCs maintained in GM represented controls. Cell proliferation was determined by MTT test. The data are mean of four to seven independent experiments performed in triplicate. Results are presented as a relative proliferation index where the baseline proliferation level of control PBMCs cultures was set at 1 $* p<0.05, * * p<0.01$ and ${ }^{* * *} p<0.001$ compared to the corresponding controls

and PB-MSCs showed marked suppression only when there was MSCs $1 \times 10^{4} /$ well, i.e. $1: 10 \mathrm{MSCs} / \mathrm{PBMCs}$ ratio.

Further on, we investigated the influence of different MSCs on MLR triggered by allogeneic antigens and results shown in Fig. 1C confirmed that all MSC populations suppressed the proliferation of PBMCs induced by allogeneic PBMCs, with AT-MSCs exhibiting the most significant inhibitory effect. Although all cell populations inhibited the MLR in a MSC concentration-dependent manner, the effects varied regarding the cell concentration, i.e. the MSC/ effector cell ratio, at which higher levels of suppression were observed. Namely, for the AT-MSCs and UC-MSCs, significant inhibition was detected with both MSC concentrations used, for DP-MSCs a more prominent effect was seen at the $1: 100 \mathrm{MSC} / \mathrm{effector}$ cell ratio, whereas PB-MSCs exhibited significant effect when there was a $1: 10 \mathrm{MSC} / \mathrm{effector}$ cell ratio.

To assess the effects of MSC-secreted bioactive molecules, we next investigated the capacity of their conditioned medium (CM) to affect either the spontaneous or mitogen-induced proliferation of allogeneic PBMCs, as well as the MLR reaction. As shown in Fig. 2, CM derived from DP-MSCs, PB-MSCs and UC-MSCs, considerably suppressed the proliferation of allogeneic PBMC (Fig. 2A), the PHA-mediated proliferation (Fig. 2B) and the MLR (Fig. 2C). On the other hand, although the MLR was effectively inhibited (Fig. 2C), the AT-MSCs-derived $\mathrm{CM}$ failed to suppress the baseline proliferation capacity of the PBMCs (Fig. 2A), as well as their PHA-induced 
responses (Fig. 2B), most probably due to marked individual variations within the effects detected for different PBMCs donors.

\section{Analysis of immunomodulatory related genes expression in different MSC populations}

The expression of genes related to MSCs immunomodulatory functions was next investigated and compared. Beside their constitutive expression profiles in the MSC populations studied, transcript levels of HLA-A, HLA-G, HLA-DR, IL-6, TGF- $\beta$, COX2 and IDO- 1 were also analyzed following the IFN- $\gamma$ and TNF- $\alpha$ stimulation, since the proinflammatory microenvironment, including these two cytokines, has been implicated in the initiation of the MSCs immunomodulatory phenotype (Fig. 3).

Regarding the members of the HLA family, the constitutive expression of both classical HLA-A class I and non-classical HLA-G5 class I molecules, as well as the
HLA-DR, was not detected in PB-MSCs, UC-MSCs and DP-MSCs, while low levels of HLA-A and HLA-DR mRNA were detected only for AT-MSCs. As for the basal gene expression of the molecules related to the paracrine effects of MSCs, a low level of IDO-1 transcript was determined only in AT-MSCs, whereas AT-MSCs, PB-MSCs and UC-MSCs displayed similar low expression levels for the TGF- $\beta$ mRNA. Concerning the IL- 6 and COX2 marked expression of their transcript levels was demonstrated for the AT-MSCS and DP-MSCs.

Further on, the gene expression in MSCs was determined after cytokine treatment and as shown in Fig. 3, the effects of the two proinflammatory cytokines used, differed between the MSC populations. In AT-MSCs, in response to IFN- $\gamma$ and TNF- $\alpha$, either alone or combined, an augmented expression of almost all tested genes (HLA-A, HLA-DR, HLA-G, COX2, IDO-1, IL- 6 and TGF- $\beta$ ) was determined. It is worth mentioning that IFN- $\gamma$ and TNF- $\alpha$
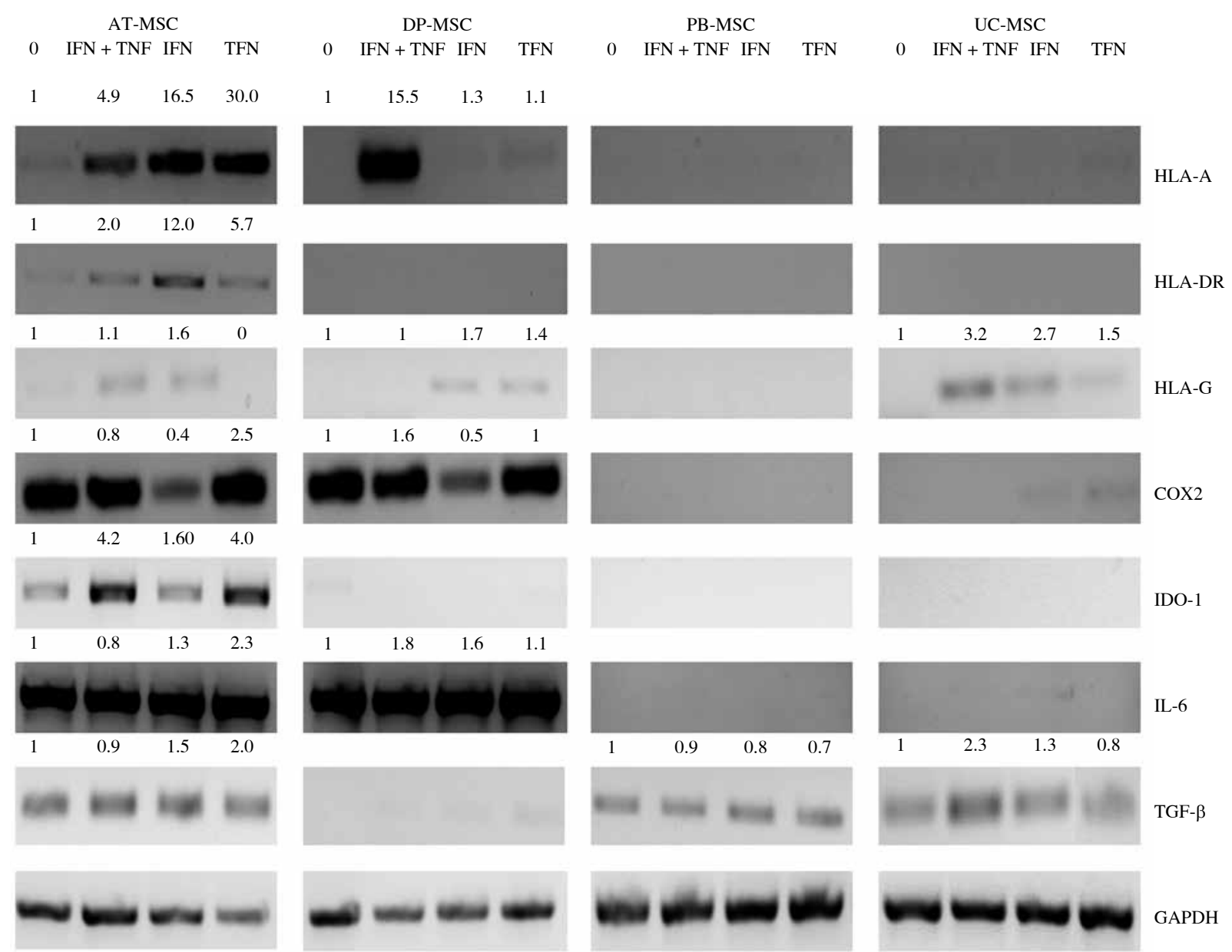

TGF- $\beta$
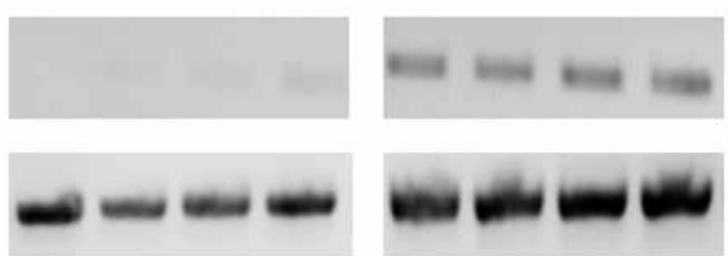

GAPDH

Fig. 3. Constitutive and IFN- $\gamma$ and/or TNF- $\alpha$ modulated gene expression of molecules related to immunomodulatory capacity in AT-MSCs, DP-MSCs, PB-MSCs, and UC-MSCs. MSCs were cultivated in GM with or without IFN- $\gamma$ $(50 \mathrm{ng} / \mathrm{ml})$ and/or TNF- $\alpha(20 \mathrm{ng} / \mathrm{ml})$ for 7 days. Gels from the representative experiment are presented. GAPDH was used as a gel loading control (Ctrl). Numbers represent densitometry values given over control with value 1 
combined stimulation did not induce synergistic upregulation of the tested genes. As for the DP-MSCs, the opposite effect was observed, since only the combined cytokine pre-treatment increased the expression of HLA-A, HLA-G5, COX2 and IL-6. Regarding the PB-MSCs, pre-treatment with these two proinflammatory cytokines did not alter the mRNA expression of tested molecules, while in response to IFN- $\gamma$ and TNF- $\alpha$, a slightly stimulated expression of HLA-G molecule and TGF- $\beta$ was determined in UC-MSCs.

\section{Discussion}

In our laboratory MSCs from adipose tissue, dental pulp of deciduous teeth, peripheral blood and Wharton's jelly of umbilical cord were recently isolated and characterized exhibiting typical MSC morphology, clonogenic potential, immunophenotype and multipotent capacity to differentiate along mesenchymal lineages (osteogenic, chondrogenic, adipogenic and myogenic) in vitro [10, 11]. It is known that MSCs isolated from different tissues have similar but not identical capacity to modulate immune response. There are studies in which immunomodulatory properties of MSCs isolated from different tissues were directly compared [17-20], but because of a great variety in applied experimental procedures it is still difficult to know which of these MSCs are the most suitable for a specific therapeutic approach. Our results showed that types of MSCs analyzed in this study differed in both, capacity to modulate proliferation of allogeneic PBMCs and, constitutive or induced (by proinflammatory cytokines) expression of several genes related to the immunomodulatory properties of MSCs.

We showed, as expected, that in the direct cell culture all MSCs types inhibited the proliferation of PBMCs in response to both the mitogen (PHA) and alloantigens. We performed our assays at relatively high MSCs to PBMCs ratios of $1: 10$ and $1: 100$, which according to previous data are "more physiological" than lower MSCs to responder cell ratio [21]. The inhibition of PBMCs proliferation obtained was cell-concentration dependent. Namely, for PHA-stimulated PBMCs proliferation, all MSCs types induced a more pronounced inhibitory effect at lower 1 : 10 MSCs to PBMCs ratio. Besides, for alloantigen-induced PBMCs proliferation more prominent inhibition was induced by AT-MSCs and UC-MSCs at both cell concentrations used, whereas DP-MSCs and PB-MSCs exerted an inhibitory effect only at $1: 100$ or $1: 10 \mathrm{MSCs} / \mathrm{PBMCs}$ ratios, respectively. Data that cell ratio determines whether MSCs would act as stimulators or inhibitors have been published previously [9, 22].

Regarding the allostimulatory function, DP-MSCs, PB-MCSs, and UC-MSCs did not provoke alloreactivity in vitro, but in contrast to them, AT-MSCs stimulated proliferation of allogeneic PBMCs, but only at the
$1: 10$ AT-MSCs to PBMCs ratio. Other authors have also demonstrated that MSCs are able to provoke alloimmune response both in vitro [23] and in vivo [24]. Furthermore, the allostimulatory function previously demonstrated for bone marrow MSCs was shown to be dependent on cell-tocell contact, but not on the soluble factor(s), as it was not abolished when metabolic activity of MSCs was prevented by pretreatment with cross-linking fixatives [23]. In addition, the allostimulatory function of MSCs demonstrated in that study was related to the constitutive membrane expression of HLA-DR molecule. However, the AT-MSCs used in our study constitutively express HLA-DR mRNA but not the membrane HLA-DR protein (our submitted manuscript), being in accordance with reports that HLA-DR molecules are habitually not expressed as surface markers unless MSCs are stimulated/primed within the inflammatory milieu $[25,26]$. Nevertheless, to better understand the mode of action of AT-MSCs on PBMCs proliferation, further studies should be performed to determine if increased AT-MSCs-stimulated proliferation of PBMCs was the result of stimulated proliferation of regulatory $\mathrm{T}$ cells as previously shown [27].

Immunomodulatory activity of MSCs could be mediated by direct cell contact or by secreted molecules $[18,28$, 29] and which mechanisms predominate is highly dependent on the applied experimental design. There are several articles demonstrating opposite effects of constitutively secreted molecules by MSCs on proliferation of allogeneic lymphocytes $[2,15,17]$. Our results showed that CM of confluent, unstimulated MSCs also differentially modulate proliferation of PBMCs. Namely, CM derived from DP-MSCs, PB-MSCs, and UC-MSCs considerably suppressed the proliferation of allogeneic PBMC, as well as the proliferation of PBMCs in response to the mitogen and alloantigens. On the other hand, AT-MSCs' culture supernatant exerted different immunomodulatory properties, as it effectively inhibited MLR, but had no effect on baseline proliferation capacity and PHA-stimulated proliferation of PBMCs. The observed inhibitory effect of CM derived from DP-MSCs, PB-MSCs, and UC-MSCs on proliferation of PBMCs could be a consequence of induced apoptosis or cell cycle arrest. However, further studies should address this issue in more detail. On the other hand, previous data evidenced the capacity of bone marrow MSCs to support survival of unstimulated T cells [30]. Whether the same is the case for AT-MSCs whose CM did not exert an inhibitory effect on PBMCs proliferation remains to be determined.

Further on, in the present study we showed that MSCs isolated from different sources in our laboratory significantly differed in the constitutive expression of several genes related to MSCs immunomodulatory functions. All tested MSC types shared a common negative expression of HLA-G5, while the constitutive gene expression of HLA-A and HLA-DR was evidenced only for AT-MSCs. 
Our results showing that all tested MSCs did not express constitutively one of the main mediators of immunosuppression, HLA-G5, were unexpected, since previously published data evidenced the expression of HLA-G5 in MSCs from different tissue sources [31]. As for the molecules related to the paracrine effects of MSCs, a marked expression of IL-6 and COX2 mRNA was demonstrated for the AT-MSCs and DP-MSCs, a low level of IDO-1 transcript only in AT-MSCs, whereas AT-MSCs, PBMSCs and UC-MSCs displayed similar low expression levels for the TGF- $\beta$ mRNA.

Since the proinflammatory microenvironment has been involved in the initiation of the immunomodulatory phenotype, we also analyzed the gene expression in MSCs following stimulation with proinflammatory cytokines, such as IFN- $\gamma$ and TNF- $\alpha$. Our results showed that the effects of these proinflammatory cytokines differed between the MSC types. Namely, in response to IFN- $\gamma$ and TNF- $\alpha$, either alone or combined, an increased expression of almost all tested genes (HLA-A, HLA-DR, HLA-G, COX2, IDO-1, IL- 6 and TGF- $\beta$ ) was determined in AT-MSCs. However, we observed that IFN- $\gamma$ and TNF- $\alpha$ combined stimulation did not induce synergistic upregulation of the tested genes in these cells, while for the DP-MSCs the opposite effect was demonstrated as only the combined cytokine pre-treatment increased the expression of HLA-A, COX2 and IL-6. On the other hand, IFN- $\gamma$ and TNF- $\alpha$ did not alter the expression of tested genes in PB-MSCs, while slightly stimulated the expression of HLA-G and TGF- $\beta$ in UC-MSCs.

Previous studies demonstrated that IFN- $\gamma$ and TNF- $\alpha$ not only differentially regulate the expression of various immunomodulatory factors by human MSCs, but also that these pro-inflammatory cytokines differentially affect the immune properties of different tissue-derived MSCs [32, 33]. According to our knowledge, a suppressive effect of the combination of TNF- $\alpha$ and IFN- $\gamma$ on the expression of genes related to immunomodulatory capacity of MSCs has not been shown. Moreover, it was shown that the combination of these cytokines increased the expression of IDO-1 in bone-marrow, adipose tissue, cord blood and Wharton's jelly MSCs [34].

Although we and other research groups showed that MSCs isolated from different tissues varied in their capacity to modulate in vitro proliferative response of allogeneic PBMCs, we cannot conclude that their different function and gene-expression profiles are entirely a consequence of their tissue origin. Indeed, in any tissue MSCs are not necessary phenotypically and functionally homogenous [35-37], and applied isolation procedures could result in isolation of different MSCs subpopulation even from the same tissues. Also, influence of donors' age and health on properties of isolated MSCs cannot be neglected [38], as well as impact of further cultivation and propagation $[12,13]$. Besides, differences in immunomodulatory ca- pacity of MSCs even from the same tissue origin could be a consequence of an experimental design applied in vitro, including the reciprocal influence on MSCs by the PBMC used in the assays, since their membrane or secreted molecules were also shown to affect the MSCs function [6]. Even more, it has been recently shown that MSCs can sense local microenvironment signals and in accordance with them promote optimal immune response, which can be either stimulatory or inhibitory depending on current specific needs of an organism [6].

In general, our results confirmed that MSCs from different tissues have immunomodulatory properties, as proliferation of PBMCs in response to mitogen or alloantigens was suppressed by all tested MSCs in cell-concentration-dependent manner. In addition, data obtained using CM MSCs indicated the involvement of soluble molecules produced by MSCs in inhibition of PBMCs proliferation. However, variations in the suppression level of allogeneic PBMCs proliferation observed for different types of MSCs, as well as marked heterogeneity in the expression of genes related to immunomodulation, emphasize the importance of investigating the intrinsic molecular mechanisms underlying MSCs immunomodulatory activity in order to provide insights for defining an optimal MSC candidate for cell-base immunotherapy.

This work has been supported by Grant No. 175062 from the Ministry of Education, Science and Technological Development of the Republic of Serbia. The authors are thankful to Mrs. Snežana Marković for her excellent technical assistance.

\section{References}

1. Augello A, Kurth TB, De Bari C (2010): Mesenchymal stem cells: a perspective from in vitro cultures to in vivo migration and niches. Eur Cell Mater 20: 121133.

2. Hass R, Kasper C, Böhm S, Jacobs R (2011): Different populations and sources of human mesenchymal stem cells (MSC): A comparison of adult and neonatal tissue-derived MSC. Cell Commun Signal 9: 12.

3. Friedenstein AJ, Petrakova KV, Kurolesova AI, Frolova GP (1968): Heterotopic of bone marrow. Analysis of precursor cells for osteogenic and hematopoietic tissues. Transplantation 6: 230-247.

4. Hong HS, Kim YH, Son Y (2012): Perspectives on mesenchymal stem cells: tissue repair, immune modulation, and tumor homing. Arch Pharm Res 35: 201-211.

5. Ghannam S, Bouffi C, Djouad F, et al. (2010): Immunosuppression by mesenchymal stem cells: mechanisms and clinical applications. Stem Cell Res Ther 15; 1: 2.

6. English K. Mechanisms of mesenchymal stromal cell immunomodulation. (2013): Immunol Cell Biol 91: 19-26.

7. Ryan JM, Barry F, Murphy JM, Mahon BP (2007): Interferon-gamma does not break, but promotes the immunosuppres- 
sive capacity of adult human mesenchymal stem cells. Clin Exp Immunol 149: 353-363.

8. DelaRosa O, Lombardo E, Beraza A, et al. (2009): Requirement of IFN-gamma-mediated indoleamine 2,3-dioxygenase expression in the modulation of lymphocyte proliferation by human adipose-derived stem cells. Tissue Eng Part A 15: 2795-2806.

9. Crop MJ, Baan CC, Korevaar SS, Ijzermans JN, et al. (2010): Inflammatory conditions affect gene expression and function of human adipose tissue-derived mesenchymal stem cells. Clin Exp Immunol 162: 474-486.

10. Nikolić N, Krstić A, Trivanović D, et al. (2011): Mesenchymal stem cell properties of dental pulp cells from deciduous teeth. Arch Biol Sci Belgrade 63: 933-942.

11. Trivanović D, Kocić J, Mojsilović S, et al. (2013): Mesenchymal stem cells isolated from peripheral blood and umbilical cord Wharton's jelly. Srpski arhiv za celokupno lekarstvo 141: 178-186.

12. Le Blanc K, Samuelsson H, Lönnies L, et al. (2007): Generation of immunosuppressive mesenchymal stem cells in allogeneic human serum. Transplantation 84: 1055-1059.

13. Samuelsson H, Ringdén O, Lönnies H, Le Blanc K (2009): Optimizing in vitro conditions for immunomodulation and expansion of mesenchymal stromal cells. Cytotherapy 11: 129-136.

14. Roobrouck VD, Clavel C, Jacobs SA, et al. (2011): Differentiation potential of human postnatal mesenchymal stem cells, mesoangioblasts, and multipotent adult progenitor cells reflected in their transcriptome and partially influenced by the culture conditions. Stem Cells 29: 871-882.

15. Ivanova-Todorova E, Bochev I, Dimitrov R, et al. (2012): Conditioned medium from adipose tissue-derived mesenchymal stem cells induces CD4+FOXP3+ cells and increases IL10 secretion. J Biomed Biotechnol doi: 10.1155/2012/295167.

16. Pearce NW, Sedgwick JD (1997): Enzyme immunoassays for detection of cell surfaced molecules, single cell products, and proliferation. In: Johnstone JP, Turner MW, editors. Immunochemistry 1, A practical approach, Oxford University Press: Oxford, New York, pp. 215-37.

17. Puissant B, Barreau C, Bourin P, et al. (2005): Immunomodulatory effect of human adipose tissue-derived adult stem cells: comparison with bone marrow mesenchymal stem cells. $\mathrm{Br}$ J Haematol 129: 118-129.

18. Wolbank S, Peterbauer A, Fahrner M, et al. (2007): Dose-dependent immunomodulatory effect of human stem cells from amniotic membrane: a comparison with human mesenchymal stem cells from adipose tissue. Tissue Eng 13: 1173-1183.

19. Najar M, Raicevic G, Boufker HI, et al. (2010): Mesenchymal stromal cells use PGE2 to modulate activation and proliferation of lymphocyte subsets: Combined comparison of adipose tissue, Wharton's Jelly and bone marrow sources: Prostaglandin E2 plays a key role in the immunosuppressive properties of adipose and bone marrow tissue-derived mesenchymal stromal cells. Exp Cell Res 316: 3109-3123.

20. Yańez R, Oviedo A, Aldea M, et al. (2010): Prostaglandin E2 plays a key role in the immunosuppressive properties of adipose and bone marrow tissue-derived mesenchymal stromal cells. Exp Cell Res 316: 3109-3123.

21. Rasmusson I, Le Blanc K, Sundberg B, Ringdén O (2007): Mesenchymal stem cells stimulate antibody secretion in human B cells. Scand J Immunol 65: 336-343.

22. Najar M, Rouas R, Raicevic G, et al. (2009): Mesenchymal stromal cells promote or suppress the proliferation of T lym- phocytes from cord blood and peripheral blood: the importance of low cell ratio and role of interleukin-6. Cytotherapy 11: 570-583.

23. Potian JA, Aviv H, Ponzio NM, et al. (2003): Veto-like activity of mesenchymal stem cells: functional discrimination between cellular responses to alloantigens and recall antigens. J Immunol 17: 3426-3434.

24. Fibbe WE, Nauta AJ, Roelofs H (2007): Modulation of immune responses by mesenchymal stem cells. Ann N Y Acad Sci 1106: $272-278$

25. Dominici M, Le Blanc K, Mueller I, et al. (2006): Minimal criteria for defining multipotent mesenchymal stromal cells. The International Society for Cellular Therapy position statement. Cytotherapy 8: 315-317.

26. Najar M, Raicevic G, Fayyad-Kazan H, et al. (2012): Immune-realeted antigens, surface molecules and regulatory factors in human-derived mesenchymal stromal cells: the expression and impact of inflammatory priming. Stem Cell Rev 8: 1188-1198.

27. Crop MJ, Baan CC, Korevaar SS, et al. (2010): Human adipose tissue-derived mesenchymal stem cells induce explosive T-cell proliferation. Stem Cells Dev 19: 1843-1853.

28. Nasef A, Chapel A, Mazurier C, et al. (2007): Identification of IL-10 and TGF-beta transcripts involved in the inhibition of T-lymphocyte proliferation during cell contact with human mesenchymal stem cells. Gene Expr 13: 217-226.

29. Wang M, Yang Y, Yang D, et al. (2009): The immunomodulatory activity of human umbilical cord blood-derived mesenchymal stem cells in vitro. Immunology 126: 220-32.

30. Benvenuto F, Ferrari S, Gerdoni E, et al. (2007): Human mesenchymal stem cells promote survival of T cells in a quiescent state. Stem Cells 25: 1753-1760.

31. Lee JM, Jung J, Lee HJ, et al. (2012): Comparison of immunomodulatory effects of placenta mesenchymal stem cells with bone marrow and adipose mesenchymal stem cells. Int Immunopharmacol 13: 219-224.

32. Prasanna SJ, Gopalakrishnan D, Shankar SR, Vasandan AB (2010): Pro-inflammatory cytokines, IFNgamma and TNFalpha, influence immune properties of human bone marrow and Wharton jelly mesenchymal stem cells differentially. PLoS One doi: 10.1371/journal.pone.0009016.

33. Hemeda H, Jakob M, Ludwig AK, et al. (2010): Interferon-gamma and tumor necrosis factor-alpha differentially affect cytokine expression and migration properties of mesenchymal stem cells. Stem Cells Dev 19: 693-706.

34. Yoo KH, Jang IK, Lee MW, et al. (2009): Comparison of immunomodulatory properties of mesenchymal stem cells derived from adult human tissues. Cell Immunol 259: 150-156.

35. Kaltz N, Ringe J, Holzwarth C, et al. (2010): Novel markers of mesenchymal stem cells defined by genome-wide gene expression analysis of stromal cells from different sources. Exp Cell Res 316: 2609-2617.

36. Jacobs SA, Roobrouck VD, Verfaillie CM, Van Gool SW (2013): Immunological characteristics of human mesenchymal stem cells and multipotent adult progenitor cells. Immunol Cell Biol 91: 32-39.

37. Yang ZX, Han ZB, Ji YR, et al. (2013): CD106 identifies a subpopulation of mesenchymal stem cells with unique immunomodulatory properties. PLoS One doi: 10.1371/journal. pone.0059354.

38. Lazzarotto-Silva C, Binato R, Rocher BD, et al. (2009): Similar proteomic profiles of human mesenchymal stromal cells from different donors. Cytotherapy 11: 268-277. 